\title{
Survey of Antibiotic-producing Bacteria Associated with the Epidermal Mucus Layers of Rays and Skates
}

\author{
Kim B. Ritchie1,2*, Melbert Schwarz ${ }^{2,3}$, Joseph Mueller², Valeri A. Lapacek², \\ Daniel Merselis ${ }^{2,4}$, Catherine J. Walsh ${ }^{2}$ and Carl A. Luer ${ }^{2}$ \\ ${ }^{1}$ Department of Natural Sciences, University of South Carolina Beaufort, Beaufort, SC, United States, ${ }^{2}$ Mote Marine \\ Laboratory, Sarasota, FL, United States, ${ }^{3}$ Department of Biological Sciences, University of Quebec at Montreal, Montreal, \\ QC, Canada, ${ }^{4}$ Department of Biological Sciences, Florida International University, Miami, FL, United States
}

\section{OPEN ACCESS}

Edited by:

Charles W. Knapp,

University of Strathclyde,

United Kingdom

Reviewed by:

Thorsten Brinkhoff,

University of Oldenburg, Germany Julie Beth Olson,

University of Alabama, United States

${ }^{*}$ Correspondence:

Kim B. Ritchie

kritch@uscb.edu

Specialty section:

This article was submitted to Antimicrobials, Resistance

and Chemotherapy,

a section of the journal

Frontiers in Microbiology

Received: 15 August 2016

Accepted: 26 May 2017

Published: 05 July 2017

Citation:

Ritchie KB, Schwarz M, Mueller J, Lapacek VA, Merselis D, Walsh CJ

and Luer CA (2017) Survey

of Antibiotic-producing Bacteria Associated with the Epidermal Mucus

Layers of Rays and Skates.

Front. Microbiol. 8:1050.

doi: 10.3389/fmicb.2017.01050
Elasmobranchs represent a distinct group of cartilaginous fishes that harbor a remarkable ability to heal wounds rapidly and without infection. To date very little work has addressed this phenomenon although it is suggested that antibiotic capabilities associated with epidermal surfaces may be a factor. The study of benefits derived from mutualistic interactions between unicellular and multicellular organisms is a rapidly growing area of research. Here we survey and identify bacterial associates of three ray and one skate species in order to assess the potential for antibiotic production from elasmobranch associated bacteria as a novel source for new antibiotics.

Keywords: antibiotic producing bacteria, antibacterial screening, pathogens, epidermal mucus, stingray, skate, beneficial bacteria

\section{INTRODUCTION}

Marine bacteria are genetically and metabolically diverse, capable of producing a wide range of chemical compounds, and are known to establish symbioses with a range of marine organisms (Bhatnagar and Kim, 2010; Sobhana, 2015). As a result, there is a growing interest in mutually beneficial associations between microbes and their hosts (Zamioudis and Pieterse, 2012). Increasing studies illustrate that bacteria produce chemical compounds that were previously ascribed to marine hosts, providing bioactive compounds for utilization in host defenses (Chau et al., 2013; Abdelmohsen et al., 2014). The study of host-associated microbes also provides a unique avenue for the search for novel bioactive compounds that could be utilized in biomedical applications (Vasanthabharathi and Jayalakshmi, 2013).

Studies in corals have shown that antibiotic activity is present in the surface mucus of healthy corals (Ritchie, 2006) Furthermore, greater than $20 \%$ of bacteria isolated from the mucus of the elkhorn coral, Acropora palmata, demonstrated antibiotic activity against a range of pathogenic test strains and $8 \%$ were specifically active against a pathogen that causes disease in this species (Ritchie, 2006). These results suggest that organisms may derive some of their immunity from probiotic bacteria associated with their surface mucus layers.

Aqueous protein extracts of the epidermal mucus layers of several finfishes have been investigated as a source of innate immunity and have demonstrated broad-spectrum antibiotic activity (John and Patterson, 2011). Similar extracts from the mucus layers of Atlantic cod, (Bergsson et al., 2005) and hagfish (Subramanian et al., 2008) have also demonstrated antibiotic activity against several common infectious pathogens, further illustrating that innate immunity 
is an important immune function for aquatic organisms. As part of their normal life activities, notably associated with regular mating events, aggressive behavior, predation, and anthropogenic encounters, sharks and their skate and ray relatives routinely sustain and recover from wounds penetrating the epidermal and dermal layers of the skin (Stevens, 1974; Pratt, 1979; HoyosPadilla et al., 2013). Such traumatic wounds heal completely and apparently without infection, even when continuously exposed to an environment rich in pathogens (Towner et al., 2012; Chin et al., 2015). With the regular occurrence of fresh and well-healed wounds on stingrays (Snelson et al., 1988; Kajiura et al., 2000) it is possible that their epidermal mucus may serve an innate immune function to account for the absence of wound-related infection.

Several studies have already investigated the possibility of such an immune function and found that chemical extracts of stingray epidermal mucus display antibiotic activity. Vennila et al. (2011) demonstrated that acidic extracts from the epidermal mucus of Dasyatis sephen, the cowtail stingray, and Himantura gerrardi, the sharpnose stingray, displayed peptide derived antimicrobial action, while Conceição et al. (2012) identified protein based antibiotic activity in the epidermal mucus of Potamotrygon henlei, the bigtooth river stingray. In addition, aqueous spine extracts of Himantura imbricata, the scaly stingray, demonstrated broad spectrum antibiotic activity (Kalidasan et al., 2014). In a microbial based, human health oriented study, it was found that the epidermal mucus of the ocellate river stingray, Potamotrygon motoro, harbored a number of bacteria that were toxic to human epithelial cells (Domingos et al., 2011). However, no studies have isolated and characterized antibiotic-producing bacteria associated with the epidermal mucus layers of stingrays.

The purpose of this study was to isolate and identify antibiotic producing bacterial strains from the epidermal mucus of skates and rays that may serve a potential probiotic role against wound infections as well as provide a novel source for antibiotics. With the realization that human wound infection pathogens are adapting to existing antibiotic drugs and becoming increasingly resistant to treatment over time, the United States Department of Defense is interested in developing new antibiotic compounds to treat their wounded warriors. As a result of the recurring observation of infection-free healing in elasmobranch fishes, the data presented here are part of a study funded by the Department of Defense to establish the feasibility of identifying novel compounds from stingray epidermal mucus with potential application in treating wound infection pathogens and promoting wound healing.

\section{MATERIALS AND METHODS}

\section{Experimental Animals}

Animals used as sources of epidermal mucus included cownose ray, Rhinoptera bonasus, Atlantic devil ray, Mobula hypostoma, marine and freshwater Atlantic stingrays, Dasyatis sabina, and clearnose skate, Raja eglanteria. All marine species are inhabitants of Gulf of Mexico waters off the coast of Sarasota, FL, United States, at various times of the year. Freshwater D. sabina are permanent inhabitants in lakes near Orlando, FL, United States.

\section{Specimen Collection}

Cownose rays and devil rays were collected passively by surrounding schools in shallow water with a seine net and transferring individual rays with dip nets to an onboard live-well. Marine Atlantic stingrays were captured in shallow nearshore water using cast nets, while deeper water clearnose skates and freshwater Atlantic stingrays were collected using baited set lines. Cownose rays, devil rays, and freshwater Atlantic stingrays were sampled at time of capture and released unharmed, while marine Atlantic stingrays and clearnose skates were returned to the laboratory where samples were collected. All animals were collected following guidelines specified in Special Activities Licenses issued by the Florida Fish and Wildlife Conservation Commission. Numbers of individuals from each species are as follows: Freshwater D. sabina, $n=3$ ( 1 male; 2 females), Marine D. sabina: $n=12$ (3 males; 9 females), M. hypostoma: $n=30$ (20 males; 10 females), $R$. bonasus: $n=74$ (27 males; 47 females), R. eglanteria; $n=3$ (all female).

\section{Mucus Collection}

Epidermal mucus was sampled from individual rays and skates by sterile seawater surface rinses followed by passive scraping of the pectoral fin surfaces with a sterile scoopula and transferred to sterile culture tubes. Mucus was separated by centrifugation $\left(2,600 \times g, 30 \mathrm{~min}, 4^{\circ} \mathrm{C}\right)$ into an aqueous supernatant and a viscous pellet (Figure 1).

\section{Bacterial Isolation}

Aliquots $(100 \mu \mathrm{L})$ of freshly collected mucus pellets and aqueous supernatants were serially diluted in sterile seawater (ranging in concentration relative to the original sample from 1:10 to $1: 1,000,000)$. One hundred microliters of each dilution was plated onto marine agar (Sigma Chemical Co) with sterile $2 \mathrm{~mm}$ ColiRoller glass beads. Cultures were grown at $25^{\circ} \mathrm{C}$ for $3-$ 5 days for development of bacterial colonies. Bacterial colonies exhibiting a unique morphology were sub-cultured 3 times to purify under the same set of growth conditions. Purified isolates were cryopreserved at $-80^{\circ} \mathrm{C}$ in $25 \%$ glycerol in marine broth and stored in 96-well microtiter plate culturable libraries for antibiotic screening. Most of the mucus-associated bacteria were isolated from the mucus pellet fraction rather than the supernatant.

\section{Antibacterial Screening}

Isolated colonies were analyzed for antibacterial properties against a number of pathogenic and non-pathogenic test strains using an agar overlay assay. Purified elasmobranch bacterial libraries were inoculated onto single well plates (VWR) of $1.5 \%$ marine agar using a 96-prong well inoculator (V\&P Scientific, Inc.). Library plates were allowed to grow at $25^{\circ} \mathrm{C}$ for 2 days and were UV- irradiated for $30 \mathrm{~min}$ to prevent further growth and cross-contamination of isolates. Test strains were grown overnight at $25^{\circ} \mathrm{C}$ (marine pathogens) or $37^{\circ} \mathrm{C}$ (human 

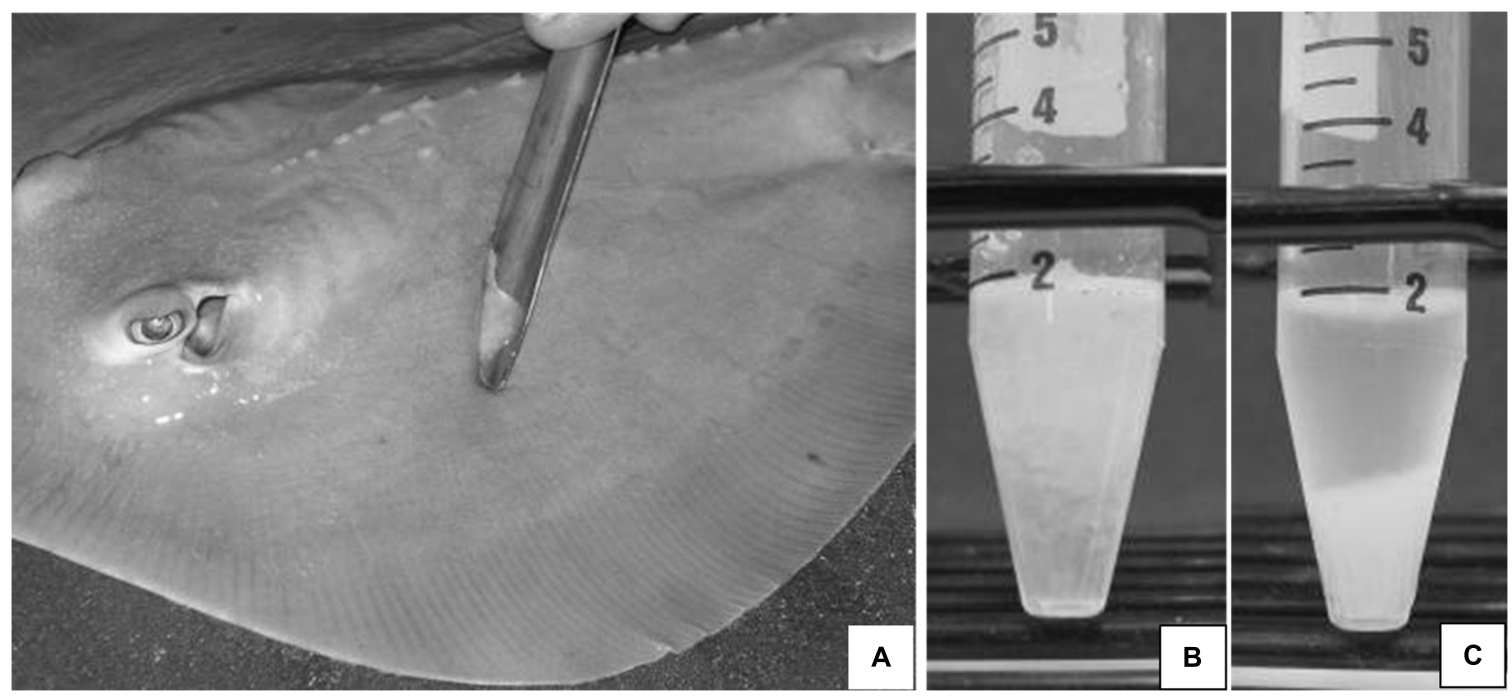

FIGURE 1 | (A) Epidermal mucus is collected by passive scraping of the pectoral fin surface of an Atlantic stingray, Dasyatis sabina with a sterile scoopula. Fresh mucus (B) can be separated into an aqueous supernatant and a mucus pellet (C) following centrifugation at $2,600 \times g$ for 20 min at $4{ }^{\circ} \mathrm{C}$.

pathogens) in $2 \mathrm{~mL}$ of strain specific culture broth and included Bacillus subtilis (ATCC $6633 \mathrm{Km}$ resistant), Enterococcus faecalis (ATCC 29212), Vancomycin-resistant Enterococcus (VRE, ATCC MP-1), Methicillin-sensitive Staphylococcus aureus (MSSA, ATCC 29213), Methicillin-resistant S. aureus (MRSA, ATCC 43300), Escherichia coli O157 Serratia marcescens PDL100 and Vibrio shilonii BAA-91. Aliquots of each broth culture were inoculated into $0.8 \%$ agar containing marine broth, Luria broth or tryptic soy broth, depending on the test strain. Elasmobranch library plates were overlaid with approximately $10 \mathrm{~mL}$ of inoculated agar. Following overnight growth, plates were analyzed for antibacterial activity and zones of inhibition were measured in millimeters.

\section{Antibacterial Compound Characterization (Blood Agar and Proteinase K Assays)}

A subset of antibiotic-producing isolates, chosen based on an ability to inhibits the growth of a varying range of pathogens as well as their stability upon repeated subculturing, was further characterized by testing for blood lysis potential and production of antimicrobial peptides. Eleven $R$. bonasus isolates displaying broad spectrum antibacterial activity in the initial screening process were inoculated onto $1.5 \%$ marine agar plates containing $5 \%$ sheep blood by volume. Following overnight growth, these strains were analyzed for the ability to lyse red blood cells by identifying and measuring zones of hemolysis (clearing zones) in millimeters. The antibacterial compounds produced by these isolates were further characterized by testing antibacterial activity in the presence of Proteinase K. Overlays were carried out as described with the addition of $100 \mu \mathrm{g} / \mathrm{mL}$ of Proteinase $\mathrm{K}$ to the test strain-appropriate $0.8 \%$ agar. Following overnight growth, zones of pathogen growth inhibition were identified and measured in millimeters. Results from this assay were then compared to initial overlay assays to determine which strains retained antibacterial properties in the presence of Proteinase K.

\section{Phylogenetic Characterization of Bacterial Strains}

DNA was extracted from pure cultures of all antibiotic-producing isolates from stingrays using a Power Soil DNA Extraction Kit (Mo Bio, Inc.). The 16S rRNA genes were amplified using a 25 $\mathrm{uL}$ polymerase chain reaction (PCR) mixture as follows: $12.5 \mu \mathrm{L}$ of Taq master mix (Qiagen), $1 \mu \mathrm{L}$ of $0.5 \mu \mathrm{g} / \mathrm{mL}$ bovine serum albumin, $8.5 \mu \mathrm{L}$ of molecular grade water, $1.0 \mu \mathrm{L}$ (50 ng) of template DNA and $1 \mu \mathrm{L}(10 \mu \mathrm{M})$ each of forward oligonucleotide primer U9F ( $5^{\prime}$-GAGTTTGATYMTGGCTC) and reverse primer U1502R (5'-GYTACCTTGTTACGACTT; Weidner et al., 1996). Cycling conditions included an initial denaturation at $94^{\circ} \mathrm{C}$ for $120 \mathrm{~s}$ followed by 35 rounds of $94^{\circ} \mathrm{C}$ for $80 \mathrm{~s}, 54^{\circ} \mathrm{C}$ for $60 \mathrm{~s}$, and $72^{\circ} \mathrm{C}$ for $90 \mathrm{~s}$ with a final $72^{\circ} \mathrm{C}$ extension for $180 \mathrm{~s}$. PCR products were identified via $1 \%$ agarose gel electrophoresis, visualized on an AlphaImager 3300, and purified using a Qiagen PCR purification kit (Qiagen, Inc). DNA from purified PCR samples was sequenced at the UIUC Core Sequencing Facility, University of Illinois, Urbana-Champaign. Consensus sequences of forward and reverse strands generated through sequencing were analyzed using the GenBank Basic Local Alignment Search Tool (BLAST) to determine percent similarity to other strains in the international bacteria sequence database (Altschul et al., 1997).

\section{Phylogenetic Tree of Antibiotic-Producing Bacteria}

A phylogenetic tree was constructed using QIIME (Caporaso et al., 2010b). Consensus sequences for each strain found in Table 2, as well as four reference sequences from GenBank 
(Pseudoalteromonas tunicata strain D2: Z2552, B. circulans: FJ581445, V. coralliilyticus: HM771346, and Photobacterium halotolerans: AY551089) were aligned with each other with the function align_seqs.py in QIIME using the PyNAST method (Caporaso et al., 2010a). Following the initial alignment, the QIIME function filter_alignment.py was used to remove gaps in the alignment shared by all sequences. Using this alignment, the phylogeny was created using FastTree 2 (Price et al., 2010), the default method utilized by the QIIME function make_phylogeny.py. The FastTree method is an approximately maximum-likelihood method in which a skeleton tree is created, using a neighbor-joining algorithm, to gain an initial topography which is subsequently improved using maximum-likelihood rearrangements. The resulting phylogeny is unrooted by default. The phylogenetic tree was visualized in $\mathrm{R}$ using the package ggtree (Yu et al., 2016).

\section{GenBank Accession Numbers}

$16 \mathrm{~S}$ rRNA gene sequences generated were entered into the GenBank world-wide database (NCBI) under accession numbers KP713443-KP713670.

\section{Ethics Statement}

This study was carried out in strict accordance with the recommendations in the Guide for the Care and Use of Laboratory Animals of the National Institutes of Health and outlined in Mote Marine Laboratory's Animal Welfare Assurance (A4219-01). All experimental protocols were approved by Mote Marine Laboratory's Institutional Animal Care and Use Committee (IACUC) and by the US Army Medical Research and Materiel Command (USAMRMC) Animal Care and Use Review Office (ACURO). All biological samples were obtained passively with minimal discomfort and no animals died or were euthanized during the project.

\section{RESULTS}

During this study, 1860 bacteria were isolated from the epidermal mucus of three stingray species and one skate species (Table 1).

TABLE 1 | Compiled stingray and skate antibacterial screening data.

\begin{tabular}{llccc}
\hline Species & $\begin{array}{l}\text { Common } \\
\text { name }\end{array}$ & $\begin{array}{c}\text { Bacterial } \\
\text { isolates }\end{array}$ & $\begin{array}{c}\text { \# Isolates with } \\
\text { antimicrobial } \\
\text { activity }\end{array}$ & $\begin{array}{c}\text { \% Showing } \\
\text { activity }\end{array}$ \\
\hline $\begin{array}{l}\text { Dasyatis } \\
\text { sabina (F) }\end{array}$ & $\begin{array}{l}\text { Atlantic } \\
\text { stingray }\end{array}$ & 96 & 9 & 9.4 \\
$\begin{array}{l}\text { Dasyatis } \\
\text { sabina (M) }\end{array}$ & $\begin{array}{l}\text { Atlantic } \\
\text { stingray }\end{array}$ & 323 & 60 & 18.6 \\
$\begin{array}{l}\text { Rhinoptera } \\
\text { bonasus }\end{array}$ & $\begin{array}{l}\text { Cownose } \\
\text { ray }\end{array}$ & 960 & 200 & 20.8 \\
$\begin{array}{l}\text { Mobula } \\
\text { hypostoma }\end{array}$ & Devil ray & 193 & 35 & 18.1 \\
$\begin{array}{l}\text { Raja } \\
\text { eglanteria }\end{array}$ & $\begin{array}{l}\text { Clearnose } \\
\text { skate }\end{array}$ & 288 & 7 & 2.4 \\
& Totals & $\mathbf{1 , 8 6 0}$ & $\mathbf{3 1 1}$ & $\mathbf{1 6 . 7}$
\end{tabular}

All bacterial isolates were screened for their ability to produce antibacterial compounds that inhibit the growth of a range of pathogenic test strains. Three hundred and eleven of these isolates demonstrated activities against one or more test strains (Table 1). Of these 311, 57 produced either broad-spectrum antibiotics or activities against VRE or MRSA only (Table 2).

\section{Antibacterial Screening}

Analysis of zones of inhibition against pathogenic test strains identified a number of bacterial isolates that displayed antibiotic properties. Examples of pathogen inhibition by mucus-associated isolates are shown in Figures 2, 3. Two hundred out of 960 (21\%) $R$. bonasus isolates, 60 of 323 (19\%) marine D. sabina isolates, 9 of 96 (9\%) of freshwater D. sabina isolates, 35 of 193 (18\%) of M. hypostoma isolates and 7 of $288(2 \%)$ of $R$. eglanteria isolates displayed inhibition against at least one test pathogen (Table 1).

\section{Phylogenetic Identification of a Subset of Total Ray Isolates}

Table 2 represents a list of representative antibiotic producing bacterial associates from ray species. Not all isolates initially tested for antibiotic activity were phylogenetically identified due to loss of viability during repeated sub-culturing and/or frozen storage, or an inability to obtain adequate template or sequence DNA. Up to $20 \%$ of antibiotic producing isolates did not survive repeated freeze thaw upon cryopreservation and are, therefore, not included in phylogenetic identification attempts. Bacteria associated with the clearnose skate, $R$. eglanteria, were not phylogenetically identified for this study due to loss of strains during long-term storage. Repeated isolates are indicated in Table 2 and were dereplicated based on identical $16 \mathrm{~S}$ rDNA sequences and antibiotic spectrum profiles.

\section{Freshwater D. sabina Bacterial Isolates}

Freshwater stingray isolates showed the greatest amount of diversity with 18 genera revealed (data not shown for nonantibiotic producing bacteria) yet had the lowest percentage of antibiotic activities among all the stingray isolates surveyed. Of 96 freshwater D. sabina bacterial isolates screened, only 9 (9\%) isolates showed antibiotic activities against one or more test strains (Table 2). Eight isolates were active against only E. coli and consisted mainly of members of the genus Pseudomonas, with one Psychrobacter and one Stenotrophomonas sp. showing similar activities against E. coli. One $B$. cereus isolate displayed broad-spectrum activity against E. coli, MRSA, MSSA and VRE (Table 2). Other non-antibacterial producing isolates making up the culturable flora of freshwater D. sabina mucus included Gordonia, Mycobacterium, Microbacterium, Caulobacter, Brevundimonas, Chryseobacterium, Staphylococcus, Psychrobacter, Nocardia, Bosea, Rhizobium, Delftia, Stenotrophomonas, Leucobacter, Acinetobacter, and Ochrobactrum spp. (data not included).

\section{Marine D. sabina Bacterial Isolates}

Of 323 marine D. sabina bacterial isolates screened, 60 (19\%) displayed antibiotic activity (Table 1), and only against B. subtilis 
TABLE 2 | List of representative bacteria isolated from the mucus of rays.

\begin{tabular}{|c|c|c|c|c|c|c|}
\hline Strain & $\mathrm{Bp}$ & $\%$ ID & Strain identification & Isolate \# & AB spectrum (ZI) & Accession \# \\
\hline $845 \mathrm{A3}$ & 780 & 99 & Microbacterium sp. & & B. subtilis (1.0) & KP713518 \\
\hline $845 \mathrm{E} 11 \mathrm{a}$ & 926 & 99 & Pseudomonas stutzeri & & E. coli (1.5) & KP713564 \\
\hline $845 \mathrm{E} 11 \mathrm{~b}$ & 902 & 99 & Pseudomonas putida & 4 & E. coli (1.5) & KP713565 \\
\hline 845E4 & 539 & 100 & Psychrobacter pacificensis & & E. coli (1.0) & KP713562 \\
\hline $845 F 2$ & 879 & 99 & Pseudomonas sp. & & E. coli $(0.5)$ & KP713566 \\
\hline
\end{tabular}

Marine Atlantic stingray, Dasyatis sabina

\begin{tabular}{|c|c|c|c|c|c|c|}
\hline 846B4 & 839 & 99 & Photobacterium damselae & 2 & B. subtilis (1.0) & KP713568 \\
\hline 846C2 & 922 & 99 & Vibrio harveyi & 2 & B. subtilis (1.0) & KP713569 \\
\hline $846 C 5$ & 939 & 99 & Photobacterium sp. & & B. subtilis (1.0) & KP713480 \\
\hline 846F8 & 1003 & 99 & Vibrio sp. & 2 & B. subtilis (1.0) & KP713572 \\
\hline
\end{tabular}

\section{Atlantic devil ray, Mobula hypostoma}

\begin{tabular}{|c|c|c|c|c|c|c|}
\hline 809A8 & 844 & 99 & Vibrio sp. & & VRE (2.0) & KP713464 \\
\hline 809B1 & 893 & 99 & Pseudoalteromonas sp. & 3 & B. subtilis (1.0) & KP713465 \\
\hline 809B6 & 962 & 99 & Pseudoalteromonas sp. & 4 & V. shilonii (1.0) & KP713468 \\
\hline 810E4 & 937 & 98 & Vibrio sp. & 10 & B. subtilis $(0.1)$ & KP713446 \\
\hline 810F6 & 976 & 99 & Alteromonas sp. & & B. subtilis $(0.5)$ & KP713449 \\
\hline $810 \mathrm{G} 1$ & 967 & 98 & Vibrio sp. & & MRSA (0.1); B. subtilis (0.2) & KP713451 \\
\hline \multicolumn{7}{|c|}{ Cownose ray, Rhinoptera bonasus } \\
\hline 803A6-1 & 880 & 99 & Exiguobacterium sp. & 3 & B. subtilis $(0.2)$ & KP713613 \\
\hline $803 \mathrm{~B} 11$ & 787 & 99 & Pseudoalteromonas sp. & & B. subtilis (1.75) & KP713582 \\
\hline 803B6-3 & 870 & 99 & Bacillus sp. & 4 & MRSA (0.3); MSSA (1.0); B. subtilis (3.5) & KP713633 \\
\hline 803B8-1 & 889 & 99 & Exiguobacterium sp. & & MRSA (0.5); MSSA (0.2); B. subtilis (0.3) & KP713634 \\
\hline 803B8-2 & 890 & 99 & Bacillus sp. & 2 & MRSA (0.5); MSSA (0.2); B. subtilis (0.3) & KP713630 \\
\hline 803B9-1 & 872 & 100 & Bacillus sp. & 2 & MRSA (0.2); B. subtilis (1.2) & KP713627 \\
\hline 803D10-2a & 881 & 99 & Lysinibacillus sp. & & MSSA (4.5); MRSA (5.0) & KP713670 \\
\hline 803D10-2c & 955 & 99 & Bacillus sp. & 3 & MSSA (4.5); MRSA (5.0) & KP713625 \\
\hline 803D5 & 860 & 99 & Halomonas sp. & & MRSA (10.5) & KP713669 \\
\hline 803D9 & 760 & 99 & Vibrio sp. & 2 & B. subtilis (1.0) & KP713585 \\
\hline 803E8 & 913 & 99 & Pseudoalteromonas sp. & & MRSA (7.5); MSSA (8.5); VRE (4.5); B. subtilis (10) & KP713479 \\
\hline $803 G 11$ & 869 & 99 & Bacillus sp. & & MRSA (6.0); MSSA (4.5); B. subtilis (7.5) & KP713626 \\
\hline $803 G 9$ & 811 & 99 & Pseudoalteromonas sp. & & B. subtilis (0.2) & KP713587 \\
\hline $803 \mathrm{H} 10$ & 787 & 99 & Pseudoalteromonas sp. & & MRSA (0.1), B. subtilis (0.1) & KP713588 \\
\hline 804C6 & 787 & 99 & Bacillus cereus & & MRSA (1.25) & KP713589 \\
\hline 804D11-1 & 837 & 100 & Bacillus sp. & & MRSA (0.1) MSSA (0.1); B. subtilis (0.2) & KP713635 \\
\hline 804D11-2 & 897 & 99 & Lysinibacillus sp. & & MRSA (0.1) MSSA (0.1); B. subtilis (0.2) & KP713636 \\
\hline 804D11-3 & 793 & 99 & Vibrio sp. & & MRSA (0.1) MSSA (0.1); B. subtilis (0.2) & KP713637 \\
\hline 804D6 & 802 & 99 & Bacillus sp. & & MRSA (1.25) & KP713591 \\
\hline 804E10 & 765 & 99 & Bacillus sp. & & MRSA (0.5); B. subtilis (0.5) & KP713592 \\
\hline 804E12 & 859 & 99 & Bacillus cereus & & MRSA (2.5) & KP713593 \\
\hline 804G3 & 909 & 99 & Pseudoalteromonas sp. & & MRSA (0.5) & KP713594 \\
\hline $804 G 9$ & 791 & 99 & Bacillus megaterium & & B. subtilis (8.0) & KP713595 \\
\hline $804 \mathrm{H} 10$ & 836 & 99 & Bacillus sp. & & MRSA (0.1), B. subtilis (0.1) & KP713476 \\
\hline $805 A 10$ & 738 & 99 & Psychrobacter celer & & B. subtilis $(0.75)$ & KP713598 \\
\hline 805A6 & 902 & 99 & Psychrobacter sp. & & B. subtilis (0.2) & KP713478 \\
\hline 805B1 & 809 & 99 & Marinobacter hydrocarbonoclasticus & & B. subtilis $(0.1)$ & KP713599 \\
\hline
\end{tabular}


TABLE 2 | Continued

\begin{tabular}{|c|c|c|c|c|c|c|}
\hline Strain & $\mathrm{Bp}$ & $\%$ ID & Strain identification & Isolate \# & AB spectrum (ZI) & Accession \# \\
\hline 805B12 & 883 & 99 & Vibrio sp. & 5 & B. subtilis $(0.5)$ & KP713600 \\
\hline $805 C 10$ & 737 & 100 & Alteromonas sp. & & B. subtilis (0.1) & KP713603 \\
\hline $805 C 12$ & 771 & 99 & Vibrio sp. & & B. subtilis (0.25) & KP713575 \\
\hline $805 C 7$ & 851 & 99 & Pseudoalteromonas sp. & & B. subtilis (1.5) & KP713601 \\
\hline 805E11 & 570 & 99 & Shewanella sp. & & B. subtilis $(0.1)$ & KP713606 \\
\hline 805E12 & 852 & 100 & Bacillus sp. & & B. subtilis (0.5) & KP713481 \\
\hline 805E7 & 860 & 99 & Pseudoalteromonas sp. & & B. subtilis (4.5) & KP713605 \\
\hline $805 F 10$ & 694 & 99 & Vibrio sp. & & B. subtilis (0.2) & KP713609 \\
\hline 805F12 & 845 & 99 & Bacillus sp. & & B. subtilis $(0.1)$ & KP713576 \\
\hline $805 F 4$ & 817 & 100 & Marinobacter sp. & & B. subtilis (0.1) & KP713607 \\
\hline $805 \mathrm{H} 7$ & 1002 & 99 & Pseudoalteromonas sp. & 11 & B. subtilis $(0.1)$ & KP713577 \\
\hline 806B10 & 832 & 99 & Shewanella sp. & & $\operatorname{MSSA}(>10)$ & KP713485 \\
\hline 806B11 & 919 & 99 & Alteromonas sp. & & $\operatorname{MSSA}(>10)$ & KP713486 \\
\hline $806 \mathrm{~B} 12$ & 795 & 99 & Pseudoalteromonas sp. & & B. subtilis $(0.2)$ & KP713487 \\
\hline $806 C 11$ & 816 & 99 & Alteromonas sp. & & $\operatorname{MSSA}(>10)$ & KP713492 \\
\hline $806 C 12$ & 886 & 99 & Pseudoalteromonas sp. & 2 & B. subtilis (0.2) & KP713493 \\
\hline $806 \mathrm{C} 7$ & 875 & 99 & Pseudoalteromonas sp. & & B. subtilis (0.5) & KP713489 \\
\hline 806C9 & 775 & 99 & Vibrio sp. & & B. subtilis (0.2) & KP713490 \\
\hline 806E11 & 831 & 99 & Vibrio maritimus & & MSSA (0.1) & KP713618 \\
\hline $806 F 10$ & 624 & 100 & Vibrio sp. & & MRSA (0.1); B. subtilis (0.2) & KP713631 \\
\hline 807A1 & 965 & 99 & Vibrio sp. & 13 & B. subtilis (0.1) & KP713496 \\
\hline 807A6 & 875 & 99 & Vibrio parahaemolyticus & & B. subtilis $(0.1)$ & KP713499 \\
\hline 807E3 & 752 & 99 & Vibrio sp. & & B. subtilis (0.2) & KP713501 \\
\hline $807 \mathrm{H} 7$ & 851 & 99 & Vibrio sp. & & VRE (1.5) & KP713507 \\
\hline $807 \mathrm{H} 8$ & 889 & 99 & Vibrio sp. & & VRE (0.5) & KP713508 \\
\hline 808A12 & 653 & 99 & Pseudoalteromonas sp. & & MRSA (0.5); B. subtilis (0.2) & KP713457 \\
\hline 808A7 & 918 & 99 & Psychrobacter sp. & & B. subtilis (0.5) & KP713456 \\
\hline 808B1 & 918 & 99 & Pseudoalteromonas sp. & & B. subtilis $(0.3)$ & KP713482 \\
\hline $808 C 1$ & 903 & 99 & Psychrobacter sp. & & B. subtilis (0.3) & KP713458 \\
\hline 808E1 & 872 & 99 & Psychrobacter sp. & & B. subtilis (0.1) & KP713459 \\
\hline $808 F 11$ & 624 & 99 & Pseudoalteromonas sp. & & MRSA (0.2); B. subtilis (0.1) & KP713460 \\
\hline $808 G 11$ & 942 & 100 & Vibrio sp. & & B. subtilis (1.5) & KP713461 \\
\hline $808 \mathrm{H} 4$ & 950 & 99 & Alteromonas sp. & & B. subtilis $(0.1)$ & KP713462 \\
\hline $814 \mathrm{~A} 6$ & 936 & 99 & Bacillus cereus & & MRSA (1.0); MSSA (2.0) & KP713578 \\
\hline $815 \mathrm{D} 3$ & 512 & 99 & Paracoccus sp. & & E. coli $(2.0)$ & KP713579 \\
\hline $815 \mathrm{G} 7$ & 834 & 99 & Pseudoalteromonas sp. & & B. subtilis $(0.1)$ & KP713581 \\
\hline $816 C 8$ & 854 & 99 & Bacillus sp. & & MRSA (2.0) MSSA (1.0); B. subtilis (4) & KP713573 \\
\hline $823 B 6-2 c$ & 804 & 99 & Exiguobacterium sp. & & MRSA (0.3) MSSA (1.0); B. subtilis (3.5) & KP713622 \\
\hline
\end{tabular}

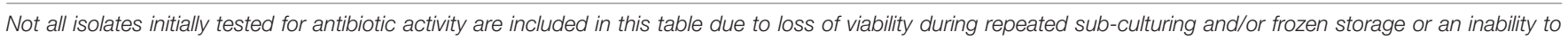

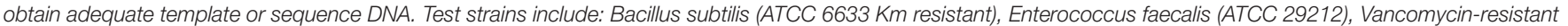

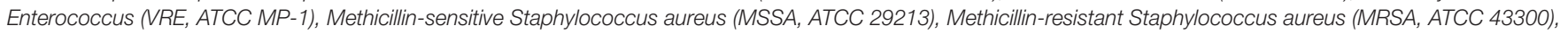

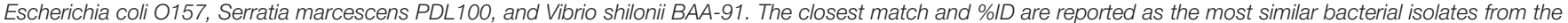

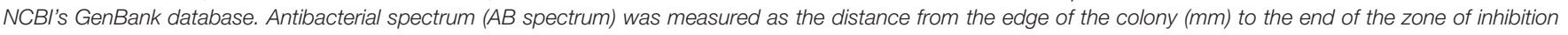

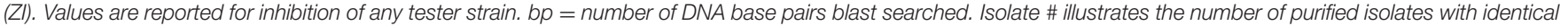
sequences and antibiotic production range/spectrum. Accession \# = Genbank accession number of the representative bacterial isolate.

(Table 2). Of eight active isolates genetically identified, 16S rDNA sequence analysis revealed 3 bacterial genera including Vibrio spp. $(n=4)$, Photobacterium spp. $(n=3)$, and a Staphylococcus sp. $(n=1)$.

\section{M. hypostoma Bacterial Isolates}

Of 193 M. hypostoma bacterial isolates screened, 35 (18\%) produced antibiotic activities against one or more test strains (Table 1). Of 20 isolates genetically identified, only three bacterial genera were revealed (Table 2). Twelve Vibrio isolates showed activities against $B$. subtilis; one showed activity against VRE; 1 showed activity against both MRSA and B. subtilis; and 1 isolate showed no antibacterial activity against any pathogenic strain tested. Ten isolates were identified as Pseudoalteromonas species with 4 isolates showing activities against B. subtilis, 4 against $V$. shilonii, and 2 showing no activities to any pathogenic strain tested. One Alteromonas species showed activity against B. subtilis only (Table 2). 


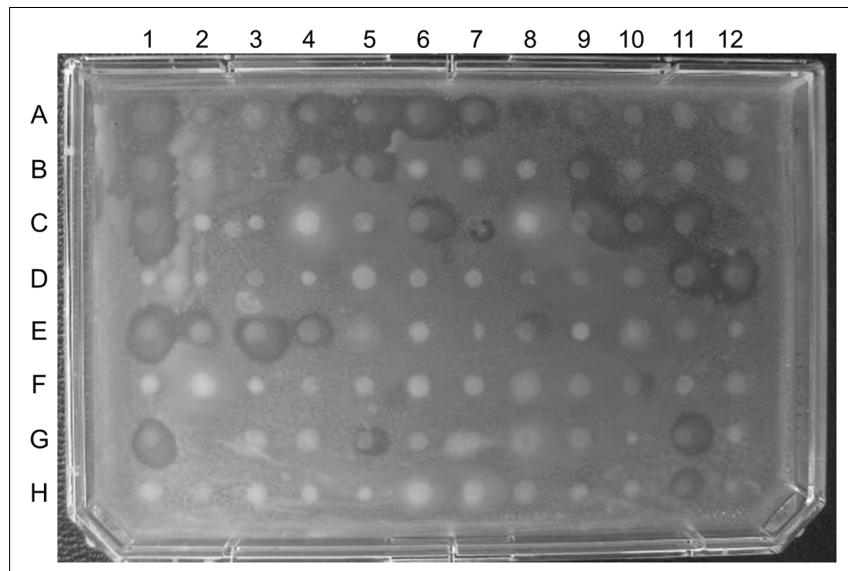

FIGURE 2 | Plate containing 96 of the 323 bacterial isolates cultured from marine Atlantic stingray (D. sabina) mucus overlaid with vancomycin-resistant enterococcus (VRE). Zones of inhibition are visible in 30 of the 96 isolates assayed on this plate.

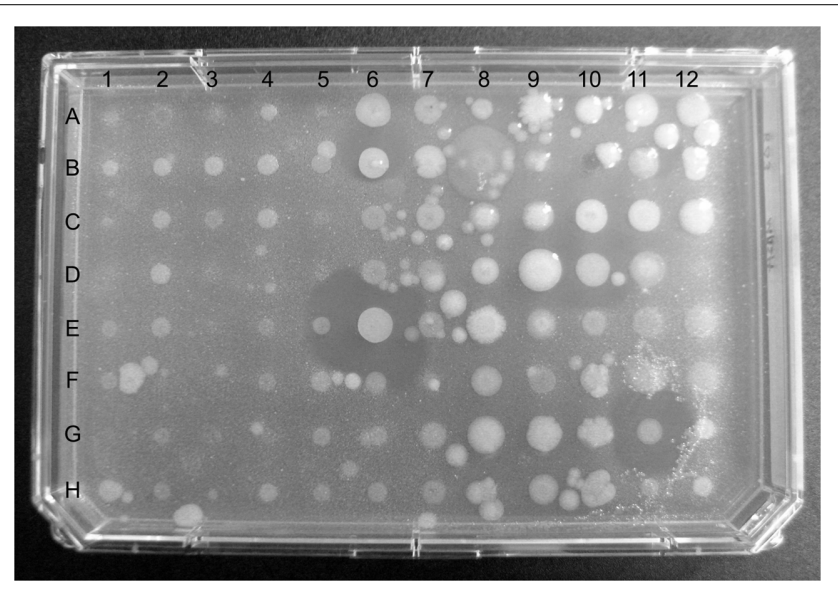

FIGURE 3 | Plate containing 96 of the 960 bacterial strains cultured from cownose ray (Rhinoptera bonasus) mucus overlaid with methicillin-resistant Staphylococcus aureus (MRSA). Diameters of zones of inhibition in wells E6 and $\mathrm{G} 11$ are $7.5 \mathrm{~mm}$ and $6.0 \mathrm{~mm}$, respectively.

\section{R. bonasus Bacterial Isolates}

Of 960 bacteria isolated and screened, 200 (21\%) were active against one or more test strain (Table 1). Eleven different bacterial genera were identified that produce antibacterial compounds, with members of the genera Bacillus, Exiguobacterium, Lysinibacillus, Vibrio, and Pseudoalteromonas displaying the broadest spectra of activity (Table 2). One Halomonas sp. (803D5) displayed antibiotic activity against MRSA with a zone of inhibition exceeding $10 \mathrm{~mm}$. Bacillus sp. 803E6, displayed large zones of inhibition against MRSA (7.5 mm), MSSA (8.5 mm), VRE $(4.5 \mathrm{~mm})$ and B. subtilis $(10 \mathrm{~mm})$ (Table 2). One Shewanella sp. (806B10) and one Alteromonas sp. (806B11) were active against MSSA with zones of inhibition over $10 \mathrm{~mm}$. Two Vibrio sp. demonstrated antibacterial activities against VRE (Table 2).

\section{Phylogenetic Tree of Antibiotic-Producing Bacteria}

To better visualize the phylogenetic relatedness of antibiotic producing strains found in this study (Table 2) a phylogenetic tree (Figure 4) was constructed. In order to address relatedness to other antibiotic-producing bacteria, we included reference sequences from $P$. tunicata strain D2: Z2552, B. circulans, FJ581445, V. coralliilyticus, HM771346, and P. halotolerans, AY551089). $R$. bonasus bacteria were the most abundant isolates genetically identified in this study and formed the basis of the three phylogenetic clusters illustrated in Figure 4. M. hypostoma isolates clustered within groups II and III and marine D. sabina clustered entirely within group II. Isolates from fresh water and marine D. sabina did not cluster together. The freshwater D. sabina isolates clustered between group I and II with a single isolate positioned within the $R$. bonasus dominated group I Bacillus isolates. This fresh water Atlantic stingray isolate, $B$. cereus $845 \mathrm{E} 9$, showed inhibition against numerous test strains (E. coli, MRSA, MSSA, VRE; Table 2). The R. bonasus isolates positioned closest to strain $845 \mathrm{E} 9$ in Figure 4 showed similar inhibitory properties against MSSA and MRSA with varying inhibitory properties against other test strains (Table 2). Alteromonas and Shewanella isolates derived from $R$. bonasus produced the strongest zones of inhibition observed against both MSSA and MRSA (heatmap, Figure 4 and Table 2).

\section{Antibacterial Compound Characterization of a Subset of Broad-Spectrum Antibacterial-Producing Isolates}

Eleven strains from M. hypostoma and R. bonasus were selected for further characterization based on activities against a range of nosocomial pathogens, as well as an ability to survive repeated subculturing and cryopreservation. Compound characterization included hemolytic activity testing and testing for activity in the presence of Proteinase K. Hemolysis tests were performed in order to identify antibiotics with potential toxicity to eukaryotic cells and proteinase $K$ tests were used to specifically determine which activities rely on the presence of small peptide antibiotics (Table 3). Seven of 9 strains tested from $R$. bonasus were found to be hemolytic. In contrast, both strains isolated from M. hypostoma did not display hemolytic activity. The activity of both M. hypostoma strains was inhibited by the presence of Proteinase $\mathrm{K}$ in antibacterial assays against MRSA and B. subtilis suggesting that the active compound is a peptide antibiotic. Antibiotic activities in 3 of $9 R$. bonasus strains were inhibited by the presence of Proteinase $K$, suggesting that the active compound(s) in these strains are peptide antibiotics.

\section{DISCUSSION}

As elasmobranchs display impressive wound healing capabilities, the intent of this study was to survey culturable ray and skate bacterial associates for antibiotic activity with the ultimate goal of identifying a new marine source for novel anti-infective compounds. Results of antibiotic overlays demonstrated that a 


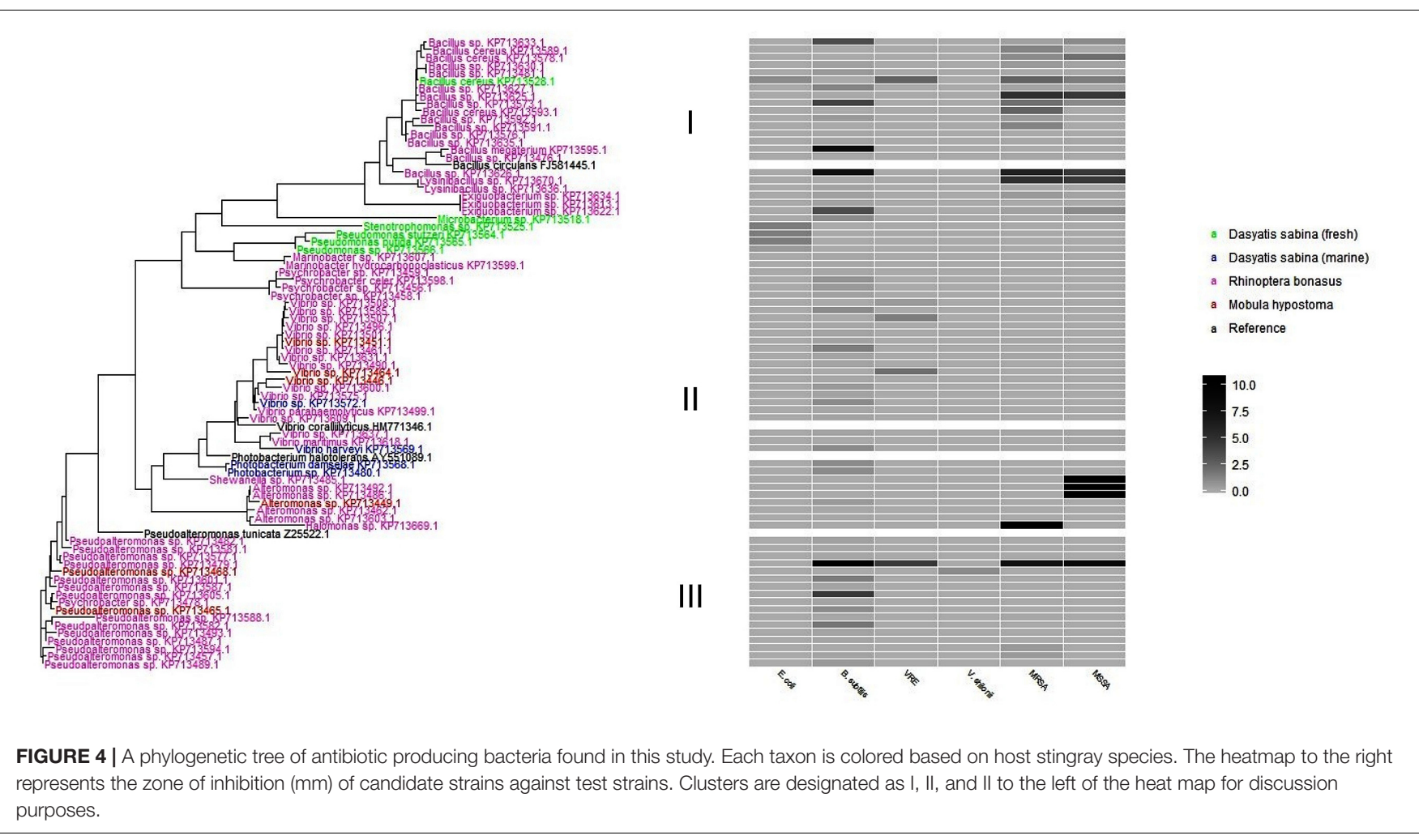

TABLE 3 | Blood agar and Proteinase K assays performed on a subset of broad-spectrum antibacterial-producing isolates.

\begin{tabular}{llll}
\hline Isolate & Zones of inhibition (mm) & Blood Agar (mm) & Proteinase K \\
\hline 803A6 & MRSA (7.5); MSSA (8.5); VRE (4.5); B. subtilis (10) & Hemolytic (2) & Uninhibited by enzyme \\
803D102C & MSSA (4.5); MRSA (5.0) & Hemolytic (0.5) & Uninhibited by enzyme \\
803E6 & MRSA (7.5); MSSA (8.5); VRE (4.5); B. subtilis (10) & Hemolytic (3) & Uninhibited by enzyme \\
803G11 & MRSA (6.0); MSSA (4.5); B. subtilis (7.5) & Not hemolytic & Uninhibited by enzyme \\
804D4 & B. subtilis (6); VRE (2.5) & Hemolytic (1) & RB \\
805D11 & B. subtilis (6.5); MRSA (2); MSSA (1.5);V. shilonii (1.0) & Hemolytic (1) & RB by enzyme \\
806E8 & B. subtilis (6.5); MRSA (2); MSSA (1.5) & Not hemolytic & Inhibited by enzyme enzyme \\
816C6 & B. subtilis (5); MRSA (2); MSSA (1) & Hemolytic (0.5) & Uninhibited by enzyme \\
816C8 & MRSA (2.0) MSSA (1.0); B. subtilis (4) & Hemolytic (2) & Uninhibited by enzyme \\
809A9 & MRSA (1); MSSA (1); VRE (1) & Not hemolytic & RB \\
809D9 & MRSA (1); MSSA (1) & Not hemolytic & RB \\
\hline
\end{tabular}

Isolates were chosen for further study based on their range of antibiotic activities against human pathogenic test strains and an ability to remain active and viable upon repeated freeze/thaw and subculturing. Zones of inhibition for antibiotic screening are indicated by measuring clearning zones in millimeters (mm). Hemolytic activity was measured on blood agar by measuring zones of clearing in millimeters $(\mathrm{mm})$. Proteinase $\mathrm{K}$ assays were utilized to determine if the active compound(s) are peptide antibiotics. The animal source is abbreviated RB for the Cownose Ray Rhinoptera bonasus and MH for the Devil Ray Mobula hypostoma.

number of mucus associated bacteria display antibiotic activity against common pathogens. In other studies, protein extracts of the stingray mucus layers have presented similar antibiotic activity (Vennila et al., 2011; Conceição et al., 2012). Collectively over $16 \%$ of bacterial isolates of the epidermal mucus from three stingray and one skate species displayed antibiotic activity against one or more pathogenic test strain. Previous studies have illustrated that roughly $3 \%$ of bacteria cultured from seawater and up to $13 \%$ of bacteria isolated from abiotic or biotic surfaces are inhibitory (Gram et al., 2010). Up to $20 \%$ of bacteria isolated from corals produce antibiotic activities (Ritchie, 2006). In the present study, roughly $3 \%$ showed either broad-spectrum antibacterial activity or activities against VRE or MRSA, only, suggesting that these isolates, and representative compounds, may be promising candidates for future drug discovery initiatives. Broad-spectrum antibiotic-producing bacterial strains isolated from stingrays, or strains active against important nosocomial pathogens (MRSA and VRE), were identified within 5 different genera including Bacillus, Vibrio, Exiguobacterium, Lysinibacterium, and Pseudoalteromonas. This information will help in targeting bacterial growth conditions for future antibiotic screens. It is important to note that some small zones of inhibition reported 
could be caused by factors other than antibiotic production, such as absence of nutrients, changes in $\mathrm{pH}$ or other variables that were not measured in this study.

Because of their ability to osmoregulate over a broad range of salinities, both marine and freshwater populations of the Atlantic stingray inhabit Florida waters. This provided a unique opportunity to consider the influence of environment on mucusassociated microbial communities in freshwater and marine D. sabina. We phylogenetically identified a large pool of total isolates from both freshwater and marine Atlantic stingrays among both antibiotic-producing and non-antibiotic-producing isolates (Table 2 and Supplementary Table 1). A predominance of Vibrio and Photobacteria spp. were associated with marine D. sabina (Supplementary Table 1). In comparison we isolated a wide range of bacterial types associated with freshwater D. sabina, including members of the genera Brevundimonas, Psychrobacter, Gordonia, Chryseobacterium, Staphylococcus, Microbacterium, Acinetobacter, Caulobacter, Mycobacterium, Bosea, Nocardia, Rhizobium, and others (Supplementary Table 1). This is the first research to our knowledge to address a comparison of $D$. sabina bacterial host specificity in fresh vs. marine waters. Other studies have shown host species-specific bacterial associations regardless of differences in environmental locations (Knowlton and Rohwer, 2003; Taylor et al., 2003). Our limited culturebased data suggest a lack of host-driven specificity in Atlantic stingrays that is likely driven by the extreme salinity differences in freshwater vs. marine environments.

Of note is the lack of overlap in antibiotic producing and non-antibiotic producing bacteria associated with freshwater $D$. sabina, where antibiotic producers fall within the genera Pseudomonas, Stenotrophomonas, Psychrobacter, and Bacillus. Representative active isolates derived from fresh water D. sabina were all active against $E$. coli with Bacillus strain $845 \mathrm{E} 9$ being the only strain with activity against gram-negative E. coli as well as gram-positive MRSA, MSSA and VRE (Table 2). In contrast, antibiotic-producing bacteria isolated from marine $D$. sabina fall within the genera Vibrio, Photobacterium, and Staphylococcus. These isolates were predominantly active against $B$. subtilis and illustrated a distinct overlap with similar isolates that did not show antibiotic-producing capabilities.

Perhaps as a reflection of their tannic acid river and lake environment, mucus from the freshwater rays is noticeably darker than mucus from marine specimens, with the majority of pigment remaining in the microbe-rich mucus pellet. In addition, in their relatively confined lake environment, freshwater $D$. sabina are exposed to anthropogenic influences of pollution from stormwater runoff and contamination from sewage (Gelsleichter et al., 2006). It is interesting to note that freshwater stingray isolates showed the greatest diversity (with 18 genera revealed among all antibiotic and non-antibiotic producing isolates genetically identified; Supplementary Table 1) and the lowest percentage of antibiotic activities among all the ray isolates surveyed (Table 1). That all nine of the isolates from freshwater ray mucus were active against $E$. coli may reflect an adaptive innate immune mechanism driven by beneficial bacterial associates. These data illustrate different bacterial community associations as well as differences in bacterial genera capable of producing antibiotic compounds associated with the epidermal mucus layers of stingrays that have adapted to dramatically different environments. Future efforts using molecular techniques will be necessary to thoroughly address differences in Atlantic stingray bacterial host specificities and potential functional redundancies of freshwater and marine bacterial associates.

The higher total number of bacteria isolated from the cownose ray, $R$. bonasus, is reflected in the higher sample size of 74 individuals from this ray species. In contrast to Atlantic stingray isolates, identification of active isolates from $R$. bonasus revealed that a majority are members of the genera Bacillus, Pseudoalteromonas, and Vibrio, with other active isolates belonging to the genera Alteromonas, Exiguobacterium, Psychrobacter, Lysinibacillus, Halomonas, Marinobacter, Shewanella, and Paracoccus. With the exception of Vibrio spp., different genera are represented among the antibiotic producing isolates from this ray species. This higher apparent diversity is likely due to the higher animal sample size. Overall, although there is apparent overlap in antibiotic producing bacterial species across all ray species analyzed, different isolates were considered unique due to host source and different colony or antibiotic spectra phenotypes and may represent bacteria in normal associations with these animals. Additional biases may include different animal capture methods. Cownose rays and devil rays were collected in natural habitats using a seine net and dip nets to access animals for sampling. While animals were rinsed with sterile seawater prior to mucus sampling, these collection techniques may have removed adhering bacteria while contributing non-associated bacteria to our isolate pools.

The phylogenetic tree shown in Figure 4 reflects stingrayderived bacterial isolates that display varying degrees of antibiotic activities. As noted above, isolates from fresh water and marine D. sabina did not cluster together. Although this study did not address total microbial community differences between hosts, this finding suggests that habitat type can drive culturable host communities even in the same host species. This is likely reflective of the long established inland freshwater versus marine habitats of these Atlantic stingray groups that influences bacterial associates. Marine Bacillus species have been shown to produce lipopeptides that are active against a range of gram positive and gram negative pathogenic test strains (Das et al., 2008) which could be a source of activity seen in our active Bacillus isolates. Pseudoalteromonas species associated with a variety of marine eukaryotes are also known to produce a range of bioactive antagonistic extracellular compounds, including antifouling agents, which may enable them to colonize surfaces (Holmström and Kjelleberg, 1999). Wietz et al. (2010) chemotyped over 300 marine Vibrionaceae and Photobacterium species revealing the presence of the previously described antibiotics, andrimid (from Vibrionaceae) and holomycin (from Photobacterium). Andrimid is a broad-spectrum peptide antibiotic originally isolated from a bacterial endosymbiont of a plant-associated arthropod (Fredenhagen et al., 1987). Holomycin also exhibits broad-spectrum activity and belongs to pyrrothine class of antibiotics that acts to interfere with RNA synthesis (Wietz et al., 2010). 
Lastly, we have sub-classified active compounds from 11 candidate antibiotic-producing bacterial strains. We selected this subset of active isolates from $R$. bonasus and M. hypostoma based on the range of test strains against which they were active, as well as their ability to retain stable activity upon repeated subculture. Proteinase $\mathrm{K}$ assays were performed in order to characterize compounds from ray associated bacterial isolates as peptide or non-peptide antibiotics (Table 3). Roughly half of the active isolates tested produce peptide antibiotics as the active compound, as assayed based on loss of antibiotic activity in the presence of proteinase K. In addition blood agar assays indicate that compounds produced by the majority of isolates tested, both peptide and non-peptide, are able to lyse blood cells (Table 3), indicating a potential toxicity to eukaryotic cells and providing another criteria from which to sub classify and prioritize active strains for future studies.

This study represents an initial search for antibiotic producing bacteria associated with ray and skate species and many isolates will be pursued for drug discovery efforts. Future studies will include molecular determination of a baseline of bacterial associates of elasmobranchs to provide a more comprehensive understanding of the stability of microbial associates across species and habitats. Future work will also contribute to an understanding of the potential role of these associates in the health and wound healing capabilities of elasmobranchs.

\section{AUTHOR CONTRIBUTIONS}

$\mathrm{KR}, \mathrm{CL}$, and CW designed the study. CL and CW collected all epidermal mucus samples. KR, MS, JM, VL, and DM carried out

\section{REFERENCES}

Abdelmohsen, U. R., Bayer, K., and Hentschel, U. (2014). Diversity, abundance and natural products of marine sponge-associated actinomycetes. Nat. Prod. Rep. 31, 381-399. doi: 10.1039/c3np70111e

Altschul, S. F., Madden, T. L., Schäffer, A. A., Zhang, J., Zhang, Z., Miller, W., et al. (1997). Gapped BLAST and PSI-BLAST: a new generation of protein database search programs. Nucleic Acids Res. 25, 3389-3402. doi: 10.1093/nar/25.17.3389

Bergsson, G., Agerberth, B., Jörnvall, H., and Gudmundsson, G. H. (2005). Isolation and identification of antimicrobial components from the epidermal mucus of Atlantic cod (Gadus morhua). FEBS J. 272, 4960-4969. doi: 10.1111/j. 1742-4658.2005.04906.x

Bhatnagar, I., and Kim, S.-K. (2010). Immense essence of excellence: marine microbial bioactive compounds. Mar. Drugs 8, 2673-2701. doi: 10.3390/ md 8102673

Caporaso, J. G., Bittinger, K., Bushman, F. D., Desantis, T. Z., Andersen, G. L., and Knight, R. (2010a). PyNAST: A flexible tool for aligning sequences to a template alignment. Bioinformatics 26, 266-267. doi: 10.1093/bioinformatics/btp636

Caporaso, J. G., Kuczynski, J., Stombaugh, J., Bittinger, K., Bushman, F. D., Costello, E. K., et al. (2010b). correspondence QIIME allows analysis of highthroughput community sequencing data Intensity normalization improves color calling in SOLiD sequencing. Nat. Publ. Gr. 7, 335-336.

Chau, R., Kalaitzis, J. A., Wood, S. A., and Neilan, B. A. (2013). Diversity and biosynthetic potential of culturable microbes associated with toxic marine animals. Mar. Drugs 11, 2695-2712. doi: 10.3390/md11082695

Chin, A., Mourier, J., and Rummer, J. L. (2015). Blacktip reef sharks (Carcharhinus melanopterus) show high capacity for wound healing and recovery following injury. Conserv. Physiol. 3:cov062. doi: 10.1093/conphys/cov062 bacterial culturing, antibiotic assays, genetic analysis, and helped prepare tables. CL prepared Figures 1-3. MS prepared Figure 4. $\mathrm{KR}, \mathrm{JM}, \mathrm{MS}$, and CL drafted the manuscript. All authors read, edited and approved the final manuscript.

\section{FUNDING}

This research was funded by a grant to CL from the United States Department of Defense through the United States. Army Medical Research and Materiel Command, Fort Detrick, Maryland, 21702-5012 (Award Number: W81XWH-11-2-0110).

\section{ACKNOWLEDGMENTS}

We thank Ben Powell, Andrew Collingwood, Ariana Agustines, Caitlin Petro, Cathleen Sullivan, Jorie Skutas, and Kari Imhof for help with lab isolations, and/or screening and analysis. We thank Steven Kembel, Diego Gil Agudelo, Daniel Conrad and two reviewers for helpful suggestions. The authors gratefully acknowledge the use of facilities at Mote Marine Laboratory (Sarasota, FL, United States).

\section{SUPPLEMENTARY MATERIAL}

The Supplementary Material for this article can be found online at: http://journal.frontiersin.org/article/10.3389/fmicb. 2017.01050/full\#supplementary-material

Conceição, K., Monteiro-Dos-Santos, J., Seibert, C., Silva, P., Marques, E., Richardson, M., et al. (2012). Potamotrygon cf. henlei stingray mucus: biochemical features of a novel antimicrobial protein. Toxicon 60, 821-829. doi: 10.1016/j.toxicon.2012.05.025

Das, P., Mukherjee, S., and Sen, R. (2008). Antimicrobial potential of a lipopeptide biosurfactant derived from a marine Bacillus circulans. J. Appl. Microbiol. 104, 1675-1684. doi: 10.1111/j.1365-2672.2007.03701.x

Domingos, M., Franzolin, M., dos Anjos, M., Franzolin, T., Barbosa Albes, R., de Andrade, G., et al. (2011). The influence of environmental bacteria in freshwater stingray wound-healing. Toxicon 58, 147-153. doi: 10.1016/j.toxicon.2011. 04.016

Fredenhagen, A., Tamura, S. Y., Kenny, P. T. M., Komura, H., Naya, Y., Nakanishi, K., et al. (1987). Andrimid, a new peptide antibiotic produced by an intracellular bacterial symbiont isolated from a brown planthopper. J. Am. Chem. Soc. 109, 4409-4411. doi: 10.1021/ja00248a055

Gelsleichter, J., Walsh, C. J., Szabo, N. J., and Rasmussen, L. E. L. (2006). Organochlorine concentrations, reproductive physiology, and immune function in unique populations of freshwater Atlantic stingrays (Dasyatis sabina) from Florida's St. Johns River. Chemosphere 63, 1506-1522. doi: 10.1016/j.chemosphere.2005.09.011

Gram, L., Melchiorsen, J., and Bruhn, J. B. (2010). Antibacterial activity of marine culturable bacteria collected from a global sampling of ocean surface waters and surface swabs of marine organisms. Mar. Biotechnol. 12, 439-451. doi: 10.1007/s10126-009-9233-y

Holmström, C., and Kjelleberg, S. (1999). Marine Pseudoalteromonas species are associated with higher organisms and produce biologically active extracellular agents. FEMS Microbiol. Ecol 30, 285-293. doi: 10.1111/j.1574-6941.1999. tb00656.x 
Hoyos-Padilla, M., Papastamatiou, Y. P., O’Sullivan, J., and Lowe, C. G. (2013). Observation of an attack by a cookiecutter shark (Isistius brasiliensis) on a white shark (Carcharodon carcharias). Pac. Sci. 67, 129-134. doi: 10.2984/67.1.10

John, S., and Patterson, J. (2011). Antibacterial activity of epidermal layer of fishes against human pathogens. Indian J. Fish. 58, 87-90.

Kajiura, S. M., Sebastian, A. P., and Tricas, T. C. (2000). Dermal bite wounds as indicators of reproductive seasonality and behaviour in the Atlantic stingray, Dasyatis sabina. . Environ. Biol. Fish. 58, 23-31. doi: 10.1023/A:100766 7108362

Kalidasan, K., Ravi, V., Sahu, S. K., Maheshwaran, M. L., and Kandasamy, K. (2014). Antimicrobial and anticoagulant activities of the spine of stingray Himantura imbricata. J. Coast. Life Med. 2, 89-93.

Knowlton, N., and Rohwer, F. (2003). Multispecies microbial mutualisms on coral reefs: the host as a habitat. Am. Nat. 162(4 Suppl.), s51-s62. doi: 10.1086/378684

Pratt, H. L. Jr. (1979). Reproduction in the blue shark, Prionace glauca. U.S. Fish. Bull. 77, 445-470.

Price, M. N., Dehal, P. S., and Arkin, A. P. (2010). FastTree 2 - approximately maximum-likelihood trees for large alignments. PLoS ONE 5:e9490. doi: 10.1371/journal.pone.0009490

Ritchie, K. B. (2006). Regulation of microbial populations by coral surface mucus and mucus-associated bacteria. Mar. Ecol. Prog. Ser. 322, 1-14. doi: 10.3354/ meps322001

Snelson, F. F., Williams-Hooper, S. E., and Schmid, T. H. (1988). Reproduction and ecology of the Atlantic stingray, Dasyatis sabina, in Florida coastal lagoons. Copeia 1988, 1729-1739. doi: 10.2307/1445395

Sobhana, K. S. (2015). Marine Microbial Diversity. Available at: http://eprints.cmfri. org.in/id/eprint/10431

Stevens, J. D. (1974). The occurrence and significance of tooth cuts on the blue shark (Prionace glauca L.) from British waters. J. Mar. Biol. Ass. U.K. 54, 373-378. doi: 10.1017/S0025315400058604

Subramanian, S., Ross, N. W., and MacKinnon, S. L. (2008). Comparison of antimicrobial activity in the epidermal mucus extracts of fish. Comp. Biochem. Physiol. 150, 85-92. doi: 10.1016/j.cbpb.2008.01.011

Taylor, M. W., Schupp, P. J., Dahllof, I., Kjelleberg, S., and Steinberg, P. D. (2003). Host specificity in marine sponge associated bacteria, and potential implications for marine microbial diversity. Environ. Microbiol. 6, 121-130. doi: 10.1046/j. 1462-2920.2003.00545.x
Towner, A., Smale, M. J., and Jewell, O. (2012). "Boat-strike wound healing in Carcharodon carcharias," in Global Perspectives on the Biology and Life History of the White Shark, ed. M. L. Domeier (Boca Raton, FL: CRC Press), 77-84. doi: 10.1201/b11532-8

Vasanthabharathi, V., and Jayalakshmi, S. (2013). Review on bioactive potential of marine microbes. Afr. J. Microbiol. Res. 7, 4683-4688. doi: 10.5897/AJMR11. 1512

Vennila, R., Kumar, K. R., Kanchana, S., Arumugam, M., Vijayalakshmi, S., and Balasubramaniam, T. (2011). Preliminary investigation on antimicrobial and proteolytic property of the epidermal mucus secretion of marine stingrays. Asian Pac. J. Trop. Biomed. 1, 239-243. doi: 10.1016/S2221-1691(11)60162-7

Weidner, S., Arnold, W., and PUhler, A. (1996). Diversity of unculLured microorganisms associated with the seagrass HaJophila stipuJacea estimated by restriction fragment length poly- morphism analysis of PCR-amplified 16S rRNA genes. Appl. Environ. Microb. 62, 766-771.

Wietz, M., Mansson, M., Gotfredsen, C. H., Larsen, T. O., and Gram, L. (2010). Antibacterial compounds from marine vibrionaceae isolated on a global expedition. Mar. Drugs 8, 2946-2960. doi: 10.3390/md8122946

Yu, G., Smith, D. K., Zhu, H., Guan, Y., and Lam, T. T. Y. (2016). ggtree: an $r$ package for visualization and annotation of phylogenetic trees with their covariates and other associated data. Methods Ecol. Evol. 8, 28-36. doi: 10.1111/ 2041-210X.12628

Zamioudis, C., and Pieterse, C. M. J. (2012). Modulation of host immunity by beneficial microbes. Mol. Plant Microbe Interact. 25, 139-150. doi: 10.1094/ MPMI-06-11-0179

Conflict of Interest Statement: The authors declare that the research was conducted in the absence of any commercial or financial relationships that could be construed as a potential conflict of interest.

Copyright (C) 2017 Ritchie, Schwarz, Mueller, Lapacek, Merselis, Walsh and Luer. This is an open-access article distributed under the terms of the Creative Commons Attribution License (CC BY). The use, distribution or reproduction in other forums is permitted, provided the original author(s) or licensor are credited and that the original publication in this journal is cited, in accordance with accepted academic practice. No use, distribution or reproduction is permitted which does not comply with these terms. 